\title{
Geschäftsmodell-getriebene Planung von Digitalisierungsmaßnahmen in Business-to-Business-Märkten - Ein Vorgehensmodell
}

\author{
Stefan Koch (D) Thomas Werani • Alexander Schauberger • \\ Manuel Mühlburger $\cdot$ Bernhard Freiseisen $\cdot$ Petra Martinek-Kuchinka
}

Eingegangen: 30. November 2017 / Angenommen: 12. Mai 2018 / Online publiziert: 29. Mai 2018 (C) Der/die Autor(en) 2018

Zusammenfassung Die Umsetzung der Digitalisierung stellt für Organisationen momentan eine der größten Herausforderungen dar und endet oft in digitalem Aktionismus. Daher entwirft dieser Beitrag ein Vorgehensmodell, das aufgrund der durchgehenden Orientierung am Geschäftsmodell und der daraus erfolgenden systematischen Ableitung und Evaluierung von Maßnahmen die zielgerichtete Planung von Digitalisierungsmaßnahmen erlaubt. Der Ansatz wird anhand des Fallbeispiels eines Industrieunternehmens illustriert und in der Umsetzung gezeigt.

Schlüsselwörter Digitale Transformation - IT-Planung · Portfoliomanagement · Geschäftsmodell

\section{Business Model Driven Planning of Digital Transformation Projects in Business-to-Business-Markets-A Process Model}

Abstract Implementing digitization is currently one of the biggest challenges for organizations and often ends in digital actionism. Therefore, this article develops a process model that allows for targeted planning of digitization measures through

\footnotetext{
S. Koch $(\bowtie) \cdot$ M. Mühlburger

Institut für Wirtschaftsinformatik - Information Engineering, Johannes Kepler Universität Linz, Altenberger Straße 69, 4040 Linz, Österreich

E-Mail: stefan.koch@jku.at

T. Werani $\cdot$ A. Schauberger

Institut für Handel, Absatz und Marketing, Abteilung Business-to-Business-Marketing, Johannes

Kepler Universität Linz, Altenberger Straße 69, 4040 Linz, Österreich

B. Freiseisen

Fronius International GmbH, Froniusplatz 1, 4600 Wels, Österreich

P. Martinek-Kuchinka

Kuchinka \& Partner GmbH, Jaxstraße 2-4, 4020 Linz, Österreich
} 
continuous orientation on the business model and systematic derivation and evaluation of measures. This will be further illustrated by the case of an industrial company.

Keywords Digital Transformation · IT-Planning · Portfolio Management · Business Model

\section{Strategische Digitalisierung und deren Umsetzung}

Digitalisierung stellt für Organisationen momentan eines der wesentlichen Themen dar. Änderungen von Geschäftsmodellen und -strategien durch digitale Technologien oder digitale Transformation (Bharadwaj et al. 2013; Matt et al. 2015; Fitzgerald et al. 2014) bedingen einen geplanten und gesteuerten Prozess. Oftmals dominieren jedoch - wie unter dem Schlagwort Industrie 4.0 - eine eingeengte Sicht oder gar ein digitaler Aktionismus.

Daher wird in diesem Beitrag eine durchgehende Orientierung am Geschäftsmodell und eine daraus erfolgende systematische Ableitung und Evaluierung von Maßnahmen vorgeschlagen und als Planungsprozess dargestellt. Damit trägt die vorliegende Arbeit auch zur generellen Diskussion bei, durch welche Methoden Anwendungen von Technologien gefunden werden können (Bergeron et al. 1991) wie beispielsweise ausgehend von organisationalen Zielen und kritischen Erfolgsfaktoren (Peffers und Gengler 2003) - und wie diese im Anschluss zu bewerten sind (Renkema und Berghout 1997; Neumeier 2017), um Wert für das Unternehmen zu generieren. Damit handelt es sich um einen Spezialfall der strategischen IT-Planung mit analogen Zielen, wie auch schon im Informationsmanagement beispielsweise von Heinrich et al. (2014) dargestellt, jedoch mit klarem Fokus auf die Geschäftsmodellperspektive in der Situationsanalyse und der weitergehenden Maßnahmenplanung zur strategischen Digitalisierung. Hess und Barthel (2017) stellen gerade auch dieses Verhältnis von Informationsmanagement und digitaler Transformation in den Mittelpunkt der Diskussion. Der Beitrag führt zuerst in das zugrundeliegende Verständnis von Geschäftsmodellen ein und stellt anschließend das Vorgehensmodell dar, welches auf Grundlage einer intensiven Literaturanalyse und fortlaufender Kontakte mit einer Vielzahl von Unternehmen entwickelt wurde. Abschließend wird anhand des Fallbeispiels eines Industrieunternehmens der Prozess illustriert und in der Umsetzung gezeigt sowie mit ersten Erfahrungen unterlegt.

\section{Geschäftsmodelle}

\subsection{Begriffsverständnis}

Weder in der Wissenschaft noch in der unternehmerischen Praxis konnte sich bis heute ein einheitliches Verständnis des Begriffs „Geschäftsmodell“ durchsetzen (Coombes und Nicholson 2013). Das liegt unter anderem auch daran, dass die Forschung zu Geschäftsmodellen stark fragmentiert und nicht eindeutig einer einzelnen Disziplin 
zuordenbar ist (Teece 2010), weshalb sich im Laufe der Zeit die unterschiedlichsten Perspektiven in voneinander unabhängigen Silos entwickelt haben (Morris et al. 2005; Zott et al. 2011).

Morris et al. (2005) haben durch eine Inhaltsanalyse von 30 Geschäftsmodell-Definitionen festgestellt, dass sich diese in drei Ebenen gliedern lassen: Auf einer rein ökonomischen Ebene definieren einige Autoren das Geschäftsmodell als Mechanismus, wie ein Unternehmen Erträge erwirtschaftet und nachhaltig Zahlungsströme sichert. Definitionen auf der operativen Ebene legen ihren Fokus auf die Unternehmensarchitektur und interne Prozesse. Auf der strategischen Ebene bildet die WertThematik den zentralen Anknüpfungspunkt. In diesem Zusammenhang definieren verschiedene Autoren (z.B. Teece 2010; Bieger und Reinhold 2011; Osterwalder und Pigneur 2011) Geschäftsmodelle als die grundsätzliche Logik, nach der ein Unternehmen Wert schafft, an die relevanten Stakeholder transferiert und über geeignete Mechanismen einen entsprechenden Wertanteil für sich selbst realisiert. Definitionen auf der strategischen Ebene beschreiben Geschäftsmodelle somit am umfassendsten, weshalb in weiterer Folge diese Perspektive zugrunde gelegt wird.

\subsection{Hauptkonfigurationen erfolgreicher Geschäftsmodelle}

Betrachtet man Geschäftsmodellansätze auf der strategischen Ebene, dann bildet zwar die Wert-Thematik den gemeinsamen Nenner, allerdings weisen diese Ansätze Unterschiede im Geschäftsmodellverständnis, den betrachteten Geschäftsmodelldimensionen und deren inhaltlicher Beschreibung auf. Werani et al. (2016) stellen ausgewählte wertbasierte Geschäftsmodellansätze gegenüber und schlagen auf Basis einer entsprechenden Bewertung einen eigenen Ansatz vor, welcher die Business Model Canvas von Osterwalder und Pigneur (2011) in wichtigen Aspekten substanziell weiterentwickelt. Auf einer strukturellen Ebene umfasst dieser Ansatz elf Geschäftsmodelldimensionen (vgl. Abb. 1), die auf der inhaltlichen Ebene durch eine umfassende Liste an Handlungsoptionen präzisiert werden.

Der strategische Geschäftsmodellansatz geht von der Prämisse aus, dass sich die grundlegende Konfiguration eines Geschäftsmodells daraus ergibt, welche Geschäftsmodelldimensionen auf der strukturellen Ebene priorisiert und welche strategischen Schwerpunkte somit verfolgt werden. In einer empirischen Studie gehen Werani et al. (2016) der Frage nach, wie erfolgreiche Geschäftsmodelle in Business-to-Business-Märkten konfiguriert sein müssen. Die Studienergebnisse zeigen, dass es vierzehn unterschiedliche Geschäftsmodellkonfigurationen gibt, durch die sich im Branchenvergleich überdurchschnittliche Umsatzrenditen erzielen lassen und die somit als erfolgreiche Geschäftsmodelle klassifiziert werden können. Von diesen vierzehn Konfigurationen werden auf Basis der Studienergebnisse sechs besonders häufig gewählt, weshalb diese auch als Hauptkonfigurationen erfolgreicher Geschäftsmodelle gesehen werden können (Werani et al. 2016). Diese Konfigurationen sind in Abb. 2 dargestellt, die folgendermaßen zu lesen ist: Eine durchgezogene Box bedeutet, dass ein Unternehmen in der betreffenden Konfiguration die jeweilige Geschäftsmodelldimension priorisiert und sich daher intensiv mit dieser befasst. Das Fehlen einer Box hingegen repräsentiert einen „Don't Care-Fall“, was bedeutet, dass es für den Erfolg der betreffenden Geschäftsmodellkonfiguration unerheblich ist, ob 


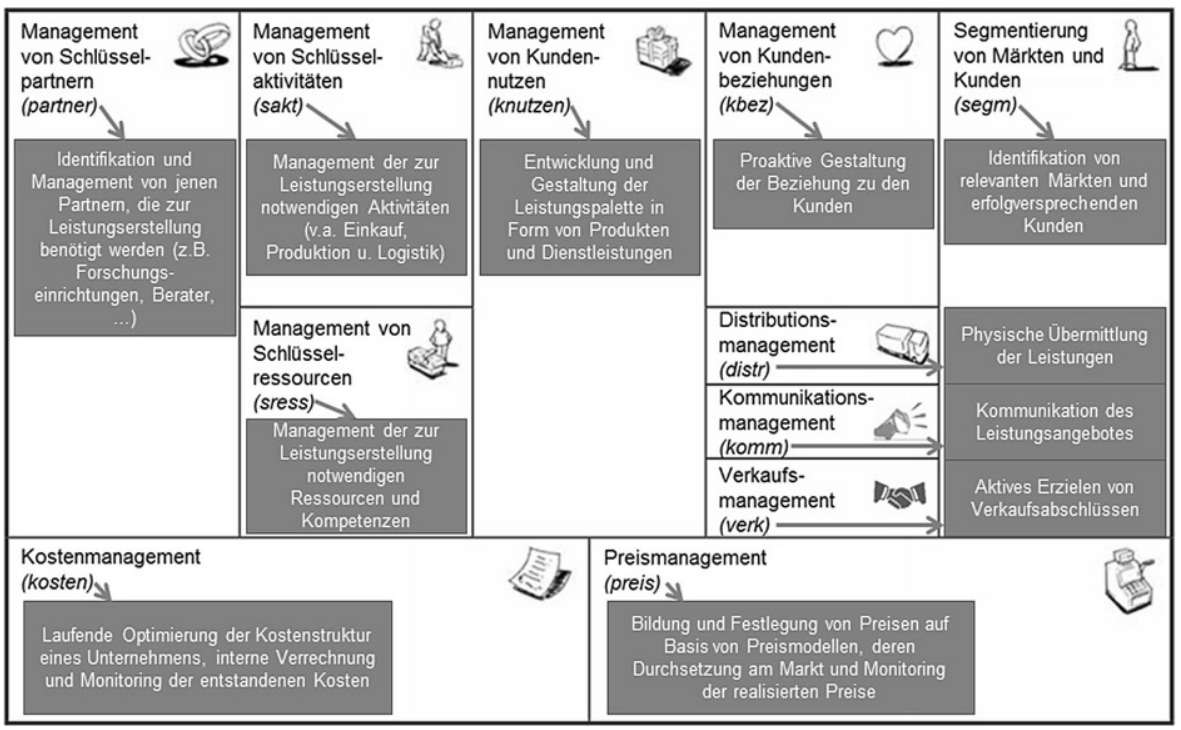

Abb. 1 Der strategische Geschäftsmodellansatz. (in Anlehnung an Osterwalder und Pigneur 2011 und Werani et al. 2016)

mit der jeweiligen Geschäftsmodelldimension eine intensive oder weniger intensive Auseinandersetzung erfolgt.

Exemplarisch bedeutet dies für die Konfiguration K1, dass sich ein Unternehmen, das diese Geschäftsmodellkonfiguration gewählt hat, intensiv mit dem Management von Kundenbeziehungen (kbez), dem Management von Kundennutzen (knutzen), dem Verkaufsmanagement (verk), dem Kommunikationsmanagement (komm), dem Preismanagement (preis) und der Segmentierung von Märkten und Kunden (segm) befasst. Die Intensität der Beschäftigung mit den verbleibenden Geschäftsmodelldimensionen ist für den Erfolg von Konfiguration K1 irrelevant. Insgesamt betrachtet verdeutlichen die Ergebnisse, dass es um erfolgreich zu sein nicht notwendig ist, sich mit allen elf Geschäftsmodelldimensionen gleich intensiv auseinanderzusetzen. Vielmehr ist es entscheidend, im Geschäftsmodell bewusst strategische Prioritäten zu setzen.

Interessant ist nun die Frage, wie die sechs Geschäftsmodellkonfigurationen aus Abb. 2 inhaltlich interpretiert werden können. Da es beim in diesem Beitrag fokussierten strategischen Geschäftsmodellansatz um die Frage der Wertschaffung, -vermittlung und -abschöpfung geht, impliziert dies unter Bezugnahme auf die Überlegungen von Treacy und Wiersema (1993), dass ein Geschäftsmodell durch die jeweils im Unternehmen verankerte Wertdisziplin geprägt wird. Die drei von Treacy und Wiersema (1993) entwickelten Wertdisziplinen - operative Exzellenz, Kundennähe und Produktführerschaft - stehen dabei jeweils für einen spezifischen Geschäftsmodellfokus mit dem Ziel, das Unternehmen von der Konkurrenz zu differenzieren und dadurch erfolgreich zu machen. Nachstehend werden aus den Überlegungen von Treacy und Wiersema (1993) die konstitutiven Elemente der drei Wertdisziplinen abgeleitet und mit den Prioritätensetzungen der sechs Geschäftsmodell- 


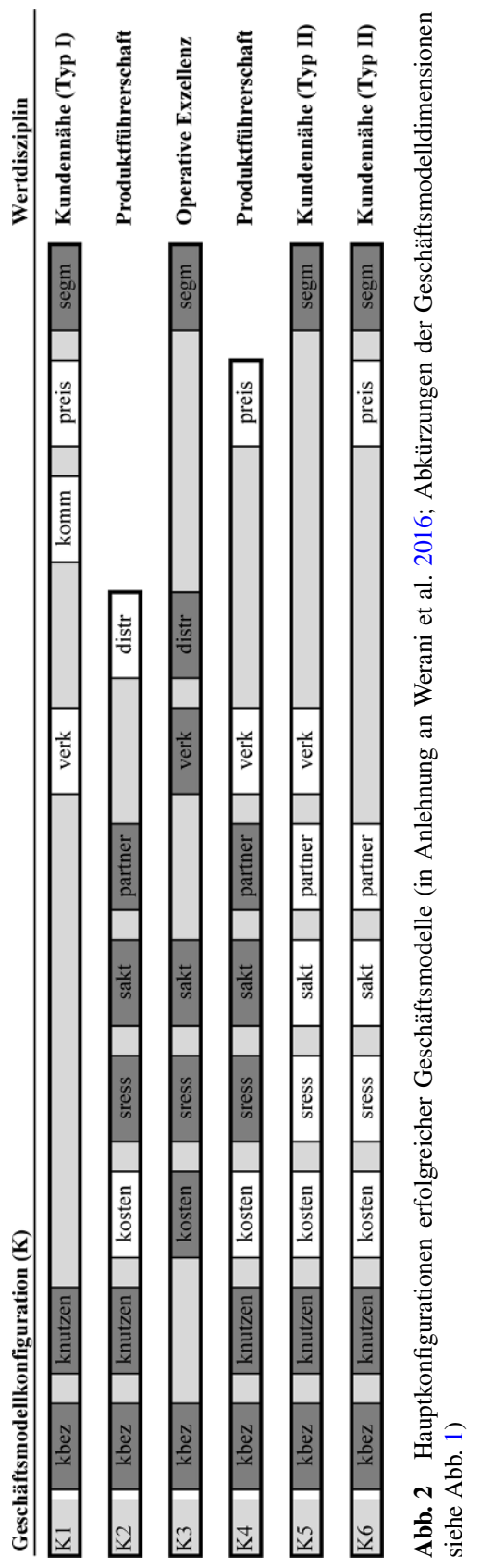


konfigurationen aus Abb. 2 abgeglichen, um zu ermitteln, ob diese Konfigurationen im Sinne von Wertdisziplinen interpretiert werden können.

Operative Exzellenz: Die erste Wertdisziplin legt ihren Fokus auf eine Minimierung der Kosten ( $\rightarrow$ kosten), eine effiziente Wertgenerierung auf Basis entsprechender Prozesse und Ressourcen $(\rightarrow$ sakt, sress) und einen effizienten Werttransfer zum Kunden ( $\rightarrow$ verk, distr). Das effizienzgetriebene Geschäftsmodell wird proaktiv auf ausgewählte Kundensegmente ausgerichtet $(\rightarrow \operatorname{segm})$, wobei der Aspekt der Qualität eine große Rolle spielt: Probleme in Prozessen, Produkten und Dienstleistungen und somit Effizienzverluste sollen möglichst vermieden werden, wobei etwa die Interaktion mit Kunden und ein Beschwerdemanagement $(\rightarrow \mathrm{kbez})$ eine wichtige Rolle spielen. Im Gegensatz zu den beiden anderen Wertdisziplinen muss die operative Exzellenz jedoch keinen Fokus auf den Kundennutzen ( $\rightarrow$ knutzen) aufweisen und hat daher häufig einen innengetriebenen Charakter. Gleicht man die genannten konstitutiven Elemente der operativen Exzellenz bzw. die damit korrespondierende Prioritätensetzung in den Geschäftsmodelldimensionen (vgl. die dunkelgrauen Boxen in Abb. 2) mit den sechs Geschäftsmodellkonfigurationen ab, so zeigt sich, dass diese Prioritätensetzung nur in K3 auftritt, welche somit die Wertdisziplin der operativen Exzellenz repräsentiert.

Kundennähe: Diese Wertdisziplin ist auf Basis der Überlegungen von Treacy und Wiersema (1993) dadurch gekennzeichnet, dass wenige Markt- bzw. Kundennischen besetzt werden $(\rightarrow$ segm) und über eine proaktive Kundenorientierung $(\rightarrow$ kbez) die gesamte Aufmerksamkeit auf die in diesen Nischen vorliegenden Kundenbedürfnisse ( $\rightarrow$ knutzen) gerichtet wird. Somit liegt ein stark beziehungsorientierter Ansatz vor. Die mit diesem Ansatz verbundene Prioritätensetzung in den Geschäftsmodelldimensionen (vgl. die dunkelgrauen Boxen in Abb. 2) spiegelt sich in den Konfigurationen K1, K5 und K6 wider, die somit die Wertdisziplin der Kundennähe repräsentieren. Wirft man einen näheren Blick auf diese Konfigurationen, so fällt auf, dass in K5 und K6 gegenüber K1 zusätzlich noch stark effizienzbezogene Geschäftsmodelldimensionen ( $\rightarrow$ kosten, sress, sakt) ins Spiel kommen. Während somit K1 der von Treacy und Wiersema (1993) beschriebenen ,idealtypischen“ Kundennähe (,Typ I“) nahe kommt, können K5 und K6 als Spielarten einer effizienzgetriebenen Kundennähe (,Typ II“) interpretiert werden.

Produktführerschaft: Die letzte Wertdisziplin ist nach Treacy und Wiersema (1993) durch überlegene Produkte und Dienstleistungen ( $\rightarrow$ knutzen) geprägt und somit stark innovationsgetrieben. Für die effiziente Entwicklung und Umsetzung von Innovationen sind geeignete Ressourcen und Prozesse $(\rightarrow$ sress, sakt) notwendig. Impulse für innovative Leistungsangebote können dabei nicht nur innerhalb des Unternehmens ( $\rightarrow$ sress), sondern auch außerhalb, etwa durch die Zusammenarbeit mit Kunden ( $\rightarrow$ kbez), entstehen. Darüber hinaus bedient sich die Produktführerschaft aber auch der Expertise externer Partner $(\rightarrow$ partner). Die für diesen Ansatz charakteristische Prioritätensetzung in den Geschäftsmodelldimensionen (vgl. die dunkelgrauen Boxen in Abb. 2) findet sich in den Konfigurationen K2 und K4, aber auch in den zuvor beschriebenen Kundennähe-Konfigurationen K5 und K6. Im 
Gegensatz zur Kundennähe setzt Produktführerschaft allerdings keinen Segmentierungsansatz $(\rightarrow$ segm) voraus, sondern stellt darauf ab, einen breiteren Markt durch Spitzenleistungen zu begeistern, welche Konkurrenzangebote aufgrund ihres Innovationsgrads obsolet machen. Somit repräsentieren letztlich nur die Konfigurationen K2 und K4 die Wertdisziplin der Produktführerschaft.

Zusammenfassend lässt sich damit konstatieren, dass erfolgreiche Geschäftsmodelle in Business-to-Business-Märkten häufig auf einer der drei grundlegenden Wertdisziplinen beruhen.

\subsection{Geschäftsmodelle und Digitalisierung}

Grundsätzlich bietet jede Geschäftsmodelldimension einen potenziellen Anknüpfungspunkt für die Digitalisierung. Allerdings ergibt sich genau daraus in der Praxis das Problem der Priorisierung, d.h. wo soll tatsächlich in einem Unternehmen digitale Transformation stattfinden? Die Studienergebnisse von Werani et al. (2016) verdeutlichen, dass die elf Geschäftsmodelldimensionen je nach gewählter Geschäftsmodellkonfiguration unterschiedliche Relevanz besitzen. Somit ist es für den Erfolg der digitaler Transformation entscheidend, dass die Digitalisierung genau an den Geschäftsmodelldimensionen ansetzt, die in erfolgreichen Geschäftsmodellen priorisiert werden. Die Geschäftsmodell- und die Digitalisierungslogik sind somit untrennbar miteinander verbunden und beugen einem digitalem Aktionismus vor.

Die divergenten Priorisierungsmuster der aufgezeigten Geschäftsmodellkonfigurationen implizieren, dass die digitale Transformation von Geschäftsmodellen nicht einem generellen Schema folgen darf. Vielmehr müssen abhängig von der gewählten Geschäftsmodellkonfiguration Geschäftsmodelle mit unterschiedlichem digitalem Fokus entwickelt werden. Hält man sich die gegenwärtige Diskussion rund um die Themenbereiche Industrie 4.0 und Smart Factories vor Augen, so wird schnell deutlich, dass sich diese Konzepte primär auf die Geschäftsmodelldimension des Managements von Schlüsselaktivitäten (Produktion, Beschaffung und Logistik) beziehen. Wird Digitalisierung mit diesen Konzepten gleichgesetzt, so führt dies dazu, dass die Digitalisierungsdiskussion in völlig falsche Bahnen gelenkt wird. Denn zum einen spielt der mit Industrie 4.0 und Smart Factories einhergehende Digitalisierungsschwerpunkt nicht notwendigerweise immer eine wichtige Rolle, wie der Fall der Kundennähe (Typ I) belegt. Und zum anderen verdeutlicht die Bandbreite der aufgezeigten Geschäftsmodellkonfigurationen, dass sich die digitale Transformation nicht an einer einzelnen Geschäftsmodelldimension festmachen lässt, sondern ein Phänomen ist, das wesentlich differenzierter gesehen werden muss.

\section{Vorgehensmodell}

\section{1 Überblick}

Die einzelnen Schritte der vorgeschlagenen Methodik und ihre Abfolge orientieren sich an etablierten Vorgehensweisen zur strategischen Planung, ausgehend von einer Situationsanalyse, gefolgt von der Zielformulierung und schließlich der Maßnah- 
menplanung (Sterrer 2014). Für den IT-Bereich wird dies beispielhaft von Heinrich et al. (2014) dargestellt. Wesentlich ist jedoch, dass durchgehend eine Geschäftsmodellperspektive, insbesondere in der Situationsanalyse und der weitergehenden Maßnahmenplanung, eingeführt und durchgehalten wird. Im Einzelnen wird die Situationsanalyse in eine Analyse des aktuellen Geschäftsmodells und eventueller Neukonfigurationen sowie eine Erhebung der IT-Landschaft aus Geschäftsmodellperspektive geteilt, welche auch parallel erfolgen können. Darauf folgend werden Digitalisierungsziele festgelegt und die Maßnahmenplanung mit einer Gap-Analyse zur Maßnahmengenerierung angestoßen. Die Methodik endet mit einer Maßnahmenpriorisierung und damit der Ableitung eines entsprechenden Maßnahmenportfolios. Eine schematische Darstellung der Methodik findet sich in Abb. 3. Der Methodik liegt ein Verständnis der strategie- bzw. geschäftsmodell-getriebenen Planung zugrunde, womit diese im Strategic Alignment Model nach Henderson und Venkatraman (1993) als Strategy Execution klassifiziert werden kann. Im Zuge der Digitalisierung wird jedenfalls das Leistungspotenzial der Informationsfunktion im Unternehmen (Heinrich et al. 2014) als hoch eingestuft, sodass der IT im Geschäftsmodell eine entsprechende Rolle eingeräumt wird.

\subsection{Festlegung der Geschäftsmodellkonfiguration}

In diesem Zusammenhang geht es zunächst um die Analyse des aktuellen Geschäftsmodells. Im Mittelpunkt steht die Frage, welche Geschäftsmodelldimensionen gegenwärtig priorisiert werden und ob bzw. welchen Zusammenhang diese Priorisierungen mit den Konfigurationen erfolgreicher Geschäftsmodelle aus der Studie von Werani et al. (2016) aufweisen. Bei diesem Schritt hilft der Business Model Analyzer ${ }^{\circledR}$, eine Software, mit der die ermittelten Priorisierungen verarbeitet und ausgewertet werden können. Auf Basis dieser Analyse ist die Entscheidung zu treffen, ob die identifizierte Geschäftsmodellkonfiguration weiterverfolgt oder aber revidiert werden soll. Für den Fall, dass gegenwärtig keine erfolgreiche Geschäftsmodellkonfiguration verfolgt wird, stellt der Business Model Analyzer ${ }^{\circledR}$ insofern eine Entscheidungshilfe zur Verfügung, als er ausgehend von der gegenwärtigen Konfiguration die nächstgelegenen erfolgreichen Konfigurationen identifiziert.

\subsection{Geschäftsmodell-orientierte Erhebung der IT-Landschaft}

Der Applikationsarchitektur wird in der Literatur und in der Praxis eine große Bedeutung zugeschrieben. Grund dafür ist deren Wahrnehmung als Bindeglied zwischen Geschäftsarchitektur und Infrastrukturarchitektur bzw. den IT-bezogenen Architekturen. Es herrscht jedoch kein einheitliches Verständnis, was den Begriff, aber auch den Inhalt dieser Teilarchitektur angeht (Durst und Bodendorf 2007; Riempp und Gieffers-Ankel 2007). Andere Begriffe, welche in diesem Kontext verwendet werden, sind Applikationsportfolio, Applikationslandschaft, Bebauungsplan (Hanschke 2016) oder Anwendungslandschaft. 


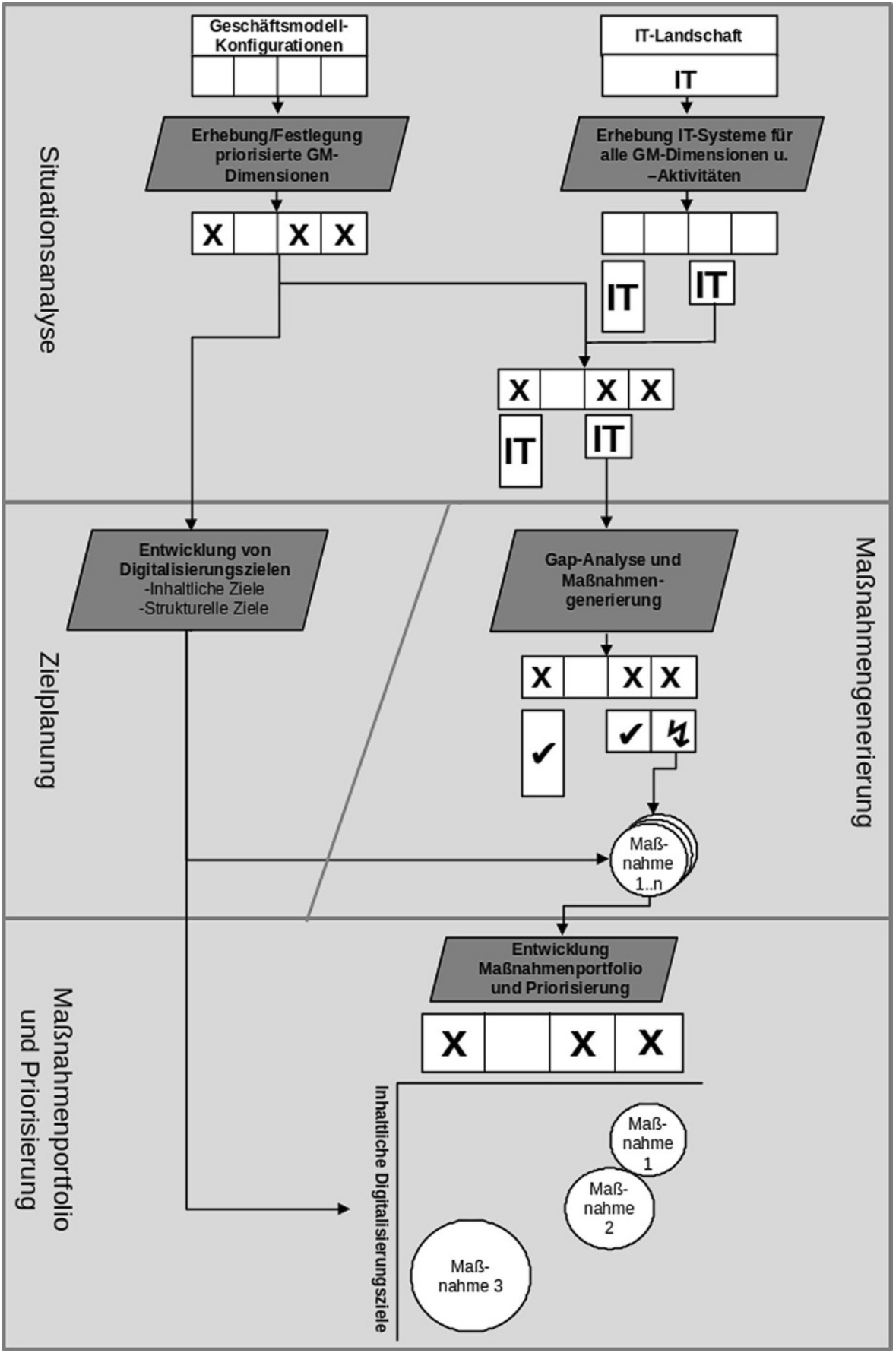

Abb. 3 Vorgehensmodell zur Planung von Digitalisierungsmaßnahmen 
Die Inhalte der Applikationsarchitektur können stark variieren. In der Praxis werden überwiegend Architekturen mit Fokus auf bzw. Gliederung nach Geschäftsprozessen abgebildet. Dies soll eine ganzheitliche Betrachtung ermöglichen, die für strategisch relevante Entscheidungen notwendig ist (Matthes 2011; Durst und Bodendorf 2007). Durch diese Miteinbringung der Geschäftsprozesse und deren Verlinkung mit den IT-bezogenen Faktoren wird ersichtlich, welche Prozesse und Bereiche die IT besonders unterstützt bzw. welche als Kernfaktor für die Effizienz erscheinen. Zudem soll aufgezeigt werden, wo große Lücken und Potenzial zur Optimierung durch die IT herrschen (Hanschke 2010). In manchen Ansätzen werden Informationsflüsse und Schnittstellen dargestellt, während andere dies vernachlässigen. Teilweise erfolgt keine weitere Gruppierung oder Gliederung, teilweise wird jedoch wieder nach Domänen oder Fachbereichen, Prozessgruppen, etc. strukturiert, wobei es sich nicht nur um fachliche Eigenschaften aus der Geschäftsarchitektur handeln muss, sondern auch IT-bezogene Eigenschaften zur Strukturierung und Gliederung herangezogen werden können (Hanschke 2010).

Im Rahmen des vorgeschlagenen Vorgehensmodells erfolgt die Erhebung der ITLandschaft anhand der in Abschn. 2.2 erläuterten Geschäftsmodelldimensionen und Handlungsoptionen nach Werani et al. (2016). Die Größe der Einträge für die einzelnen Systeme entspricht dabei den jeweiligen Kosten, welche sowohl laufende Betriebs- und Wartungskosten als auch eventuell bereits fixierte Erweiterungskosten inkludieren (siehe Abb. 4 für einen beispielhaften Auszug). Dadurch wird klar dargestellt, in welche Dimensionen Ressourcen momentan stärker investiert werden bzw. wo diese gebunden sind. Zudem wird gegenüber einer Darstellung anhand von Geschäftsprozessen die Verbindung zur strategischen Ebene verstärkt. Insbesondere wird im Vergleich auch der Blick auf die Organisationsumwelt, wie gerade auch Kunden und deren Nutzenerwartungen sowie die Beziehungen zu diesen, verstärkt, wohingegen eine interne Optimierungsperspektive nicht mehr dominant auftritt. Gerade einer Analyse basierend auf Produkten oder Fachbereichen ist zumeist eine Verankerung im momentanen Status eines Geschäftsmodells inhärent. Durch die vorgeschlagene veränderte Sichtweise wird die Entwicklung von entsprechenden Maßnahmen in den weiteren Schritten unterstützt.

\subsection{Definition von Digitalisierungszielen}

Im nächsten Schritt werden die Digitalisierungsziele festgelegt. In diesem Zusammenhang sind einerseits die inhaltlichen Ziele in Abhängigkeit von der gewählten Geschäftsmodellkonfiguration und andererseits die strukturellen Ziele zur Schaffung von IT-Strukturen bzw. Fähigkeiten zur Umsetzung der Digitalisierung zu definieren. Insbesondere die inhaltlichen Ziele spezifizieren die Implementierung des generellen Geschäftsmodells. 


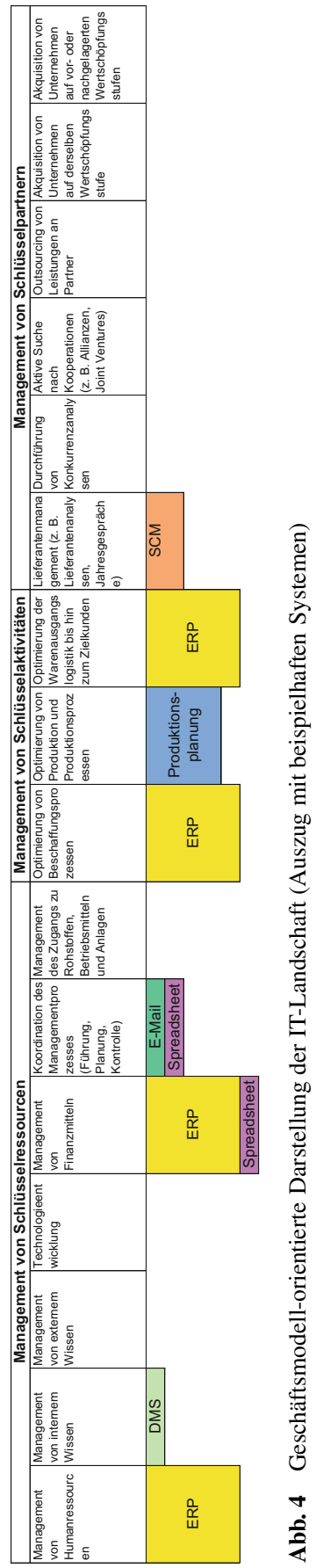




\subsubsection{Inhaltliche Ziele}

Da wie dargestellt Geschäftsmodell- und die Digitalisierungslogik untrennbar miteinander verbunden sind, muss auch bei der Formulierung inhaltlicher Digitalisierungsziele auf die gewählte Geschäftsmodellkonfiguration Bezug genommen werden. Inhaltliche Digitalisierungsziele müssen grundsätzlich unternehmensindividuell festgelegt und auf Basis einer systematischen Unternehmens- und Kundenanalyse ermittelt werden. Nachstehend sollen beispielhaft einige mit den drei grundlegenden Wertdisziplinen korrespondierende Ziele angeführt werden:

Kundennähe

- Automatisierung einer breiten und tiefen Kundendatensammlung und Generierung von Kundenprofilen

- Schaffung personalisierter digitaler Kundenschnittstellen

- Schaffung digitaler Kommunikationskanäle

- Entwicklung von Remote Services

Produktführerschaft

- Nutzung digitaler Kundendaten zur Produktoptimierung

- Virtuelle Produktentwicklung

- Digitales Wissensmanagement

- Digitaler Innovations-Workflow

Operative Exzellenz

- Digitale Steuerung von Produktionsprozessen

- Digitales Beschaffungswesen

- Entwicklung digitaler Vertriebskanäle

- Nutzung elektronischer Rekrutierungssysteme/HR-Plattformen

\subsubsection{Strukturelle Ziele}

Parallel zu den inhaltlichen sind im Unternehmen auch strukturelle Digitalisierungsziele zu definieren. Diese dienen der Schaffung von IT-Strukturen bzw. Fähigkeiten zur Umsetzung von digitaler Transformation im Unternehmen. Ein Fokus wird in diesem Punkt im Normalfall auf entsprechenden IT-Aspekten wie der Einführung von agilem Projektmanagement bzw. bimodaler IT, der Schaffung einer integrierten, skalierbaren und agilen IT-Architektur oder der Implementierung strategiegetriebener IT-Kontrolle und -Governance liegen, es können jedoch auch Ziele im Bereich der Personalentwicklung wie beispielsweise Digital Literacy (Karimi und Walter 2015; El Sawy et al. 2016) verankert werden. 


\subsection{Gap-Analyse und Maßnahmengenerierung}

Basierend auf der Priorisierung von Geschäftsmodelldimensionen kann nun die IT-Landschaft einer Gap-Analyse unterzogen werden. Ein Abgleich der momentanen Abdeckung beziehungsweise Ausrichtung inklusive finanzieller Verpflichtungen kann einen Einblick in eventuell existente Schwerpunktsetzungen erlauben, die nicht der vorgenommenen Priorisierung von Geschäftsmodelldimensionen entsprechen. Zudem werden Geschäftsmodellbereiche sichtbar, die priorisiert, jedoch momentan noch nicht entsprechend IT-seitig abgedeckt sind. Dies erlaubt die Generierung entsprechender Maßnahmen, wie die Lücken durch neue Anwendungen im Rahmen der Digitalisierung zielgerichtet geschlossen werden können und somit letztlich eine geschäftsmodellbezogene Neuausrichtung der IT-Landschaft. Die generierten Maßnahmen werden im folgenden Schritt einer Priorisierung insbesondere auch im Licht der inhaltlichen Digitalisierungsziele und damit der konkreten Geschäftsmodellimplementierung unterzogen.

\subsection{Ableitung eines Maßnahmenportfolios und Priorisierung}

Die Schaffung von Geschäftswert aus der IT ist seit Jahrzehnten ein Thema (McAfee und Brynjolfsson 2008), und ist auch heute im Kontext der digitalen Transformation relevant. Ansätze zum Gruppieren, Bewerten und zum Teil graphischen Darstellen von Anwendungen bzw. Projekten basieren auf verschiedenen Kriterien, meist unter Verwendung einer zweidimensionalen Matrix. Die am häufigsten herangezogenen Ansätze verwenden Risiko versus Nutzen oder Wert für das Unternehmen (Jeffery und Leliveld 2004). Andere Vorschläge beziehen sich auf externe versus interne Nutzeneffekte (Notowidigdo 1984) oder gruppieren in Phasen im Kundenlebenszyklus (Ives und Learmonth 1984). Tjan (2001) hat beispielsweise Passung und Durchführbarkeit als Achsen vorgeschlagen. Neumeier (2017) bevorzugt im Kontext von Digitalisierungsprojekten die Werttreiber Kundenerlebnis und Effizienz.

Im Rahmen des vorgeschlagenen Ansatzes zur Digitalisierung erfolgt die Priorisierung von Maßnahmen anhand des Beitrags zur Zielerreichung und auf Basis der priorisierten Geschäftsmodelldimensionen bzw. einer ersten groben Kosten- sowie Risikoschätzung. In einer Matrixdarstellung werden in Zeilen die inhaltlichen Digitalisierungsziele, in Spalten Geschäftsmodelldimensionen dargestellt. Die Maßnahmen werden dann in den Zellen dargestellt, und zwar für jedes Ziel sowie jede Geschäftsmodelldimension, welche davon betroffen ist. Die Größe in der Darstellung stellt eine erste Kostenschätzung dar (siehe Abb. 5 für einen beispielhaften Auszug). Eine Maßnahme, die zur Erreichung mehrerer Ziele beiträgt sowie mehrere Geschäftsmodelldimensionen abdeckt, wird höher priorisiert. Maßnahmen, welche vor Anwendung des Vorgehensmodells bereits eingeplant wurden, können ebenfalls eingetragen und - gegebenenfalls mit negativem Ergebnis - evaluiert werden.

Die entsprechend priorisierten Maßnahmen müssen in weiterer Folge einer detaillierten Analyse, insbesondere hinsichtlich Machbarkeit sowie Kosten und Nutzen unterzogen werden. Zudem sind logische Abhängigkeiten entsprechend festzustellen. Aus dieser Analyse kann unter Bezugnahme auf verfügbare Budgets schließlich eine Roadmap abgeleitet werden. 


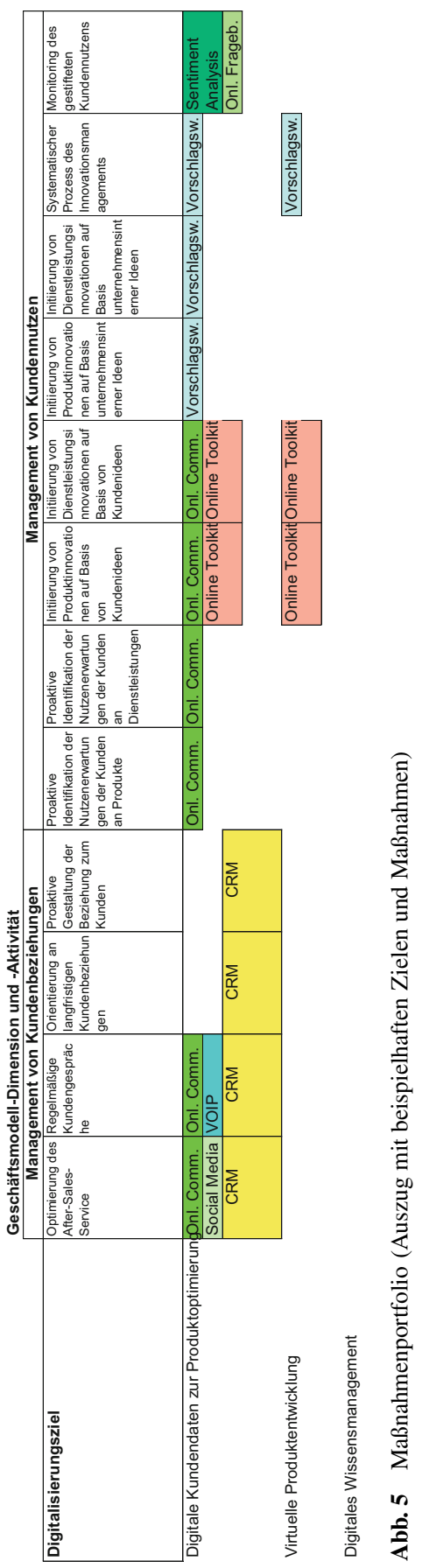




\section{Geschäftsmodellkonforme Digitalisierung - Fallbeispiel}

Fronius ist ein eigentümergeführtes österreichisches Unternehmen mit drei strategischen Geschäftseinheiten. In der Business Unit „Perfect Welding“ werden Geräte und Anlagen für das elektrische Lichtbogenschweißen von Metallen entwickelt, produziert und verkauft. Im relevanten Markt ist Fronius Perfect Welding als anerkannter Technologieführer positioniert.

Die fortschreitende Digitalisierung auf Produktebene führt im weltweiten Schweißgerätemarkt immer mehr zu einer Angleichung von technologischen Produkteigenschaften der unterschiedlichen Hersteller. Um nachhaltig im globalen Wettbewerbsumfeld bestehen zu können, war es daher erforderlich, grundlegend über eine strategische Neuausrichtung der Business Unit „Perfect Welding“ und daraus abzuleitende Projekte nachzudenken. Die Verwendung des in diesem Beitrag vorgestellten Vorgehensmodells erfolgte dabei unter Einbindung der Autoren, sodass entsprechende Eindrücke und Rückmeldungen direkt gesammelt werden konnten. Zur Festlegung der relevanten Aspekte der Neuausrichtung erfolgte in einem ersten Schritt (vgl. Abschn. 3.2) eine Reflexion der Ausgangssituation. Dazu wurden auf Basis des Business Model Analyzer ${ }^{\circledR}$-Tools die Managementprioritäten im aktuellen Geschäftsmodell mit dem Ergebnis analysiert, dass bis dato eine Produktführerschafts-Strategie verfolgt wurde. Aufgrund der skizzierten Marktund Wettbewerbssituation wurde entschieden, diese Strategie zukünftig durch eine Strategie der Kundennähe abzulösen. Als für die Business Unit strategierelevante Eckpfeiler waren somit die mit dieser Geschäftsmodellkonfiguration korrespondieren Managementprioritäten festzulegen. Unter Berücksichtigung der Studienergebnisse von Werani et al. (2016) fiel die Wahl auf die Geschäftsmodellkonfiguration K1 und somit auf die ,idealtypische“ Kundennähe, da diese Spielart mit sechs Geschäftsmodellprioritäten eine vergleichsweise geringe Managementkomplexität aufweist.

Um eine geschäftsmodell- und damit strategiekonforme Planung der Digitalisierungsmaßnahmen und Ausrichtung der IT-Landschaft in der Business Unit zu gewährleisten, müssen sich die laufenden und zukünftigen Digitalisierungsprojekte an den Geschäftsmodellprioritäten der gewählten Konfiguration K1 orientieren. Dies impliziert, dass zunächst diese Digitalisierungsprojekte mit Blick darauf zu beurteilen waren, ob und in welcher Form diese mit den zu priorisierenden Geschäftsmodelldimensionen und damit der anzustrebenden IT-Landschaft konform gehen (vgl. Abschn. 3.3 und 3.5), woraus sich eine Projekt-Neubewertung (vgl. Abschn. 3.6) ergab. Jene Projekte, welche direkt mit einer zu priorisierenden Dimension in $\mathrm{Zu}-$ sammenhang standen, wurden dabei höher bewertet, während alle anderen Vorhaben als nachrangig eingestuft wurden. Für den Fall, dass einer zu priorisierenden Geschäftsmodelldimension mehrere Projekte zugeordnet wurden, musste nochmals eine weiterführende Reihung vorgenommen werden. Diese erfolgte anhand der zwei inhaltlichen Digitalisierungsziele (vgl. Abschn. 3.4) der Business Unit: Auf Ebene der Kundenbeziehung wird das Ziel verfolgt, durch Daten ein ganzheitliches Wissen über die Kunden zu erlangen, während auf der Leistungsebene Daten ein genaues Wissen über die Produktverwendung ermöglichen sollen. Abschließend wurden alle 
intendierten Digitalisierungsprojekte hinsichtlich ihres unternehmerischen Risikos für die Geschäftseinheit bewertet, um so zu einem finalen Projekt-Set zu gelangen.

Erste Erfahrungen im Anwendungsfall Fronius bestätigen die Nützlichkeit und grundlegende Logik des entwickelten Vorgehensmodells in einer realen Organisation. Zudem konnte die Durchführbarkeit der einzelnen Verfahrensschritte mit vertretbarem Aufwand festgestellt werden. Eine finale Bewertung des Vorgehensmodells ist naturgemäß jedoch erst nach wiederholtem Einsatz in verschiedenen Unternehmen möglich.

\section{Fazit und Ausblick}

Der vorliegende Beitrag hat ein Vorgehensmodell zur strategischen Digitalisierung durch stringente Orientierung am Geschäftsmodell entworfen und liefert damit einen Beitrag sowohl zur Weiterentwicklung der betrieblichen Praxis als auch zum Verhältnis von Informationsmanagement und digitaler Transformation (Hess und Barthel 2017). Der vorgeschlagene Prozess beinhaltet zum einen eine Geschäftsmodellanalyse, zum anderen aber auch eine Analyse der IT-Landschaft, und erstreckt sich bis hin zur Maßnahmenplanung und dem Portfoliomanagement. Wie im Fallbeispiel skizziert, bewährt sich dieses Vorgehen in der Praxis und führt zu managementrelevanten Ergebnissen. In weiteren frühphasigen Anwendungen konnte zudem bereits der grundsätzliche Verfahrensnutzen bestätigt werden. Notwendig ist allerdings eine breitere Anwendung des Vorgehensmodells, um dieses im Sinne eines konstruktionsorientierten Ansatzes vollständig evaluieren und weiterentwickeln zu können. Darüber hinaus lassen sich dadurch auch wesentliche Erkenntnisse zum Zusammenhang von Geschäftsmodellen und digitaler Transformation in der Praxis gewinnen.

Funding Open access funding provided by Johannes Kepler University Linz.

Open Access Dieser Artikel wird unter der Creative Commons Namensnennung 4.0 International Lizenz (http://creativecommons.org/licenses/by/4.0/deed.de) veröffentlicht, welche die Nutzung, Vervielfältigung, Bearbeitung, Verbreitung und Wiedergabe in jeglichem Medium und Format erlaubt, sofern Sie den/die ursprünglichen Autor(en) und die Quelle ordnungsgemäß nennen, einen Link zur Creative Commons Lizenz beifügen und angeben, ob Änderungen vorgenommen wurden.

\section{Literatur}

Bergeron F, Buteau C, Raymond L (1991) Identification of strategic information systems opportunities: applying and comparing two methodologies. Mis Q 15(1):89-103

Bharadwaj A, El Sawy OA, Pavlou PA, Venkatraman N (2013) Digital business strategy: toward a next generation of insights. Mis Q 37(2):471-482

Bieger T, Reinhold S (2011) Das wertbasierte Geschäftsmodell: Ein aktualisierter Strukturierungsansatz. In: Bieger T, zu Knyphausen-Aufseß D, Krys C (Hrsg) Innovative Geschäftsmodelle. Springer, Berlin, S $14-70$

Coombes P, Nicholson J (2013) Business models and their relationship with marketing: a systematic literature review. Ind Mark Manag 42(6):656-664

Durst M, Bodendorf F (2007) Wertorientiertes Management von IT-Architekturen. Dt. Univ.-Verl., Wiesbaden 
Fitzgerald M, Kruschwitz N, Bonnet D, Welch M (2014) Embracing digital technology: a new strategic imperative. MIT Sloan Manage Rev 55(2):1-12

Hanschke I (2010) Strategic IT management. Springer, Berlin

Hanschke I (2016) Enterprise Architecture Management - einfach und effektiv: ein praktischer Leitfaden für die Einführung von EAM, 2. Aufl. Hanser, München

Heinrich LJ, Riedl R, Stelzer D (2014) Informationsmanagement: Grundlagen, Aufgaben, Methoden, 11. Aufl. De Gruyter, Oldenbourg

Henderson JC, Venkatraman H (1993) Strategic alignment: Leveraging information technology for transforming organizations. IBM Syst J 32(1):472-484

Hess T, Barthel P (2017) Wieviel digitale Transformation steckt im Informationsmanagement? Zum Zusammenspiel eines etablierten und eines neuen Managementkonzepts. HMD Prax Wirtschaftsinform 54(3):313-323

Ives B, Learmonth GP (1984) The information system as a competitive weapon. Commun Acm 27(12):1193-1201

Jeffery M, Leliveld I (2004) Best practices in IT portfolio management. MIT Sloan Manage Rev 45(3):41-49

Karimi J, Walter Z (2015) The role of dynamic capabilities in responding to digital disruption: a factorbased study of the newspaper industry. J Manage Inform Syst 32(1):39-81

Matt C, Hess T, Benlian A (2015) Digital transformation strategies. Bus Inf Syst Eng 57(5):339-343

Matthes D (2011) Enterprise architecture frameworks kompendium. Springer, Berlin

McAfee A, Brynjolfsson E (2008) Investing in the IT that makes a competitive difference. Harv Bus Rev 86(7/8):98-107

Morris M, Schindehutte M, Allen J (2005) The entrepreneur's business model: toward a unified perspective. J Bus Res 58(6):726-735

Neumeier A (2017) Wert der Digitalisierung - Erfolgreiche Auswahl von Digitalisierungsprojekten. HMD Prax Wirtschaftsinform 54(3):338-350

Notowidigdo $\mathrm{MH}$ (1984) Information systems: weapons to gain the competitive edge. Financ Executive, S 20-25

Osterwalder A, Pigneur Y (2011) Business Model Generation: Ein Handbuch für Visionäre, Spielveränderer und Herausforderer. Campus, Frankfurt am Main

Peffers K, Gengler CE (2003) How to identify new high-payoff information systems for the organization. Commun ACM 46(1):83-88

Renkema TJ, Berghout EW (1997) Methodologies for information systems investment evaluation at the proposal stage: a comparative review. Inform Softw Tech 39(1):1-13

Riempp G, Gieffers-Ankel S (2007) Application portfolio management: a decision-oriented view of enterprise architecture. Inf Syst E Bus Manag 5:359-378

El Sawy OA, Kræmmergaard P, Amsinck H, Vinther AL (2016) How LEGO built the foundations and enterprise capabilities for digital leadership. Mis Q Exec 15(2):141-166

Sterrer C (2014) Das Geheimnis erfolgreicher Projekte: Kritische Erfolgsfaktoren im Projektmanagement. Springer, Berlin

Teece D (2010) Business models, business strategy and innovation. Long Range Plann 43(2-3):172-194

Tjan AK (2001) Finally, a way to put your Internet portfolio in order. Harv Bus Rev 79(2):76-85

Treacy M, Wiersema F (1993) Customer intimacy and other value disciplines. Harv Bus Rev 71(1):84-93

Werani T, Freiseisen B, Martinek-Kuchinka P, Schauberger A (2016) How should successful business models be configured? Results from an empirical study in business-to-business markets and implications for the change of business models. J Bus Econ 86(6):579-609

Zott C, Amit R, Massa L (2011) The business model: recent developments and future research. J Manag 37:1019-1042 